\title{
Unmanned Aerial Vehicle Speed Estimation via Nonlinear Adaptive Observers
}

\author{
Khadidja Benzemrane, Giovanni L. Santosuosso and Gilney Damm
}

\begin{abstract}
In this paper the problem of the speed estimation of an Unmanned Aerial Vehicle is addressed, when acceleration, the angles and the angular speeds are available for measurement. We focus our analysis on a prototype drone - a 4 rotors helicopter robot- which is not equipped with GPS related devices and relies on the Inertial Measurement Unit (IMU) only. A global exponential solution to this open problem is provided in the framework of adaptive observation theory when exact measurements are available. A modified estimator is presented to enhance robustness in velocity estimation in the realistic case of noisy acceleration measurements.
\end{abstract}

\section{INTRODUCTION}

The problem of speed estimation has an important role in the context of vehicles control. For land moving robots, the odometry time derivative has a satisfactory performance while for large flying airships (manned or unmanned) velocity estimations can be obtained via approximate derivation of the successive measurements from GPS sensors, motivated by the small resulting errors compared to the measured variables. For fast aircrafts the standard procedure is integrating the acceleration, and coupling this result to the derivative of GPS measurements.

Two critical issues arise in an "open loop" strategy like direct acceleration integration : an unknown constant estimation error is produced even when exact acceleration measurements are available while a random drift is induced by noisy acceleration estimations. In practice, numerical integration along with measurement noise induces a very fast growing velocity measurement error. Thus the information obtained from the GPS is used to bound this error in the framework of sensor fusion technique where initial conditions for acceleration integration are provided by GPS devices. This technique provides bounded errors that are related to the GPS order of magnitude precision. The resulting errors are usually small compared to the size of airships and the distance to obstacles; In the same way, the control systems usually applied on these airships are robust enough to accept the residual disturbance on the speed estimation provided by these methods.

Unfortunately, the estimation approach above cannot be implemented on small drones less than 1 meter wide and

K.Benzemrane is with Laboratoire IBISC - CNRS/Université d'Evry Val d'Essonne, 40 rue du Pelvoux 91020 Evry Cedex, France khadidja.benzemrane@ibisc. fr

G. Santosuosso is with Dipartimento di Ingegneria Elettronica, Universitá di Roma Tor Vergata, Via del Politecnico 1, 00133 Rome, Italy santosuossoling.uniroma2. it

G. Damm is with Laboratoire IBISC - CNRS/Université d'Evry Val d'Essonne, 40 rue du Pelvoux 91020 Evry Cedex, France gilney.dammeibisc. fr flying at low speed that we consider in this note. This issue is even more relevant in indoors or simply urban applications. As a matter of fact, errors induced by a GPS system may reach many meters; a technical solution would be the use of a D-GPS system. These systems are known as centimetric GPS and have a precision of some centimeters, but they also are very expensive and low-range operating equipment. Doppler measurements coupled with GPS can actually increase velocity accuracy estimation. In this paper we focus our attention on Inertial Measurement Units only which represent a feasible choice : they are easily available, low cost devices. More important, they have a low size, limited weight, and provide very good airship measurements of accelerations, angles and angular speeds.

However, the information provided by these devices needs to be processed to yield reliable velocity estimates. The latter quantity is necessary for an efficient drone control design, since most literature about small flying robots control assumes that at least the speeds are available (see [5], [6], [7], [8]); This is motivated by the fact that is hardly possible for a human pilot (inboard or in tele-operation) to regulate a drone by giving references and trajectories as inputs based on the sole acceleration measurement. Notice that in the general case, the reliable estimation of the speed vector is still an open problem.

Motivated by previous arguments, in this note we describe a novel observation strategy that solves the problem of the speed estimation of an Unmanned Aerial Vehicle, when the angular acceleration, the angles and the angular speeds are available for measurement. We focus our analysis on a prototype drone - a 4 rotors helicopter robot shown in Fig. 1 - produced to operate in an urban environment at the Laboratoire IBISC - CNRS, Universite d'Evry, which is not equipped with GPS related devices and relies on the Inertial Measurement Unit only. We provide a global exponential solution to this open problem in the light of classical adaptive estimation theory when exact measurements are available. A modified estimator is presented to enhance robustness in velocity estimation in the realistic case of noisy acceleration measurements. The observation strategy final goal is an efficient robot's stabilization control. The simulation results obtained on the model of the real drone -even in the presence of noise- are very promising, and will be soon implemented on the physical prototype. 


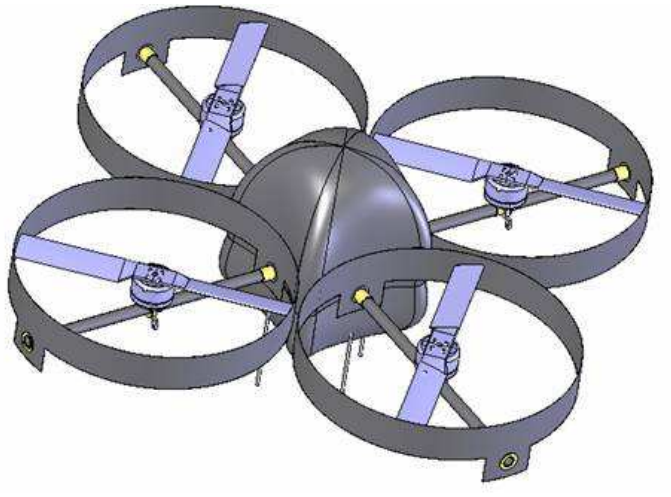

Fig. 1. Prototype Drone

In section II we formulate the problem statement and describe the drone model. We then use this information in section III where the main results are shown about the observer design procedure as well as a sketch of estimation convergence proof. In section $\mathrm{V}$ we report some simulations that illustrate the observer performance and stress the influence of some parameters on the observer behavior. Finally in section VI we conclude the note with few remarks along with an outline of our future research on this topic.

\section{PROBLEM FORMULATION}

The motion of a four rotor aerial robot can be described by the following equations (see [4]).

$$
\begin{aligned}
\dot{x}= & \cos \theta \cos \psi u \\
& +(\sin \phi \sin \theta \cos \psi-\cos \phi \sin \psi) v \\
& +(\cos \phi \sin \theta \cos \psi+\sin \phi \sin \psi) w \\
\dot{y}= & \cos \theta \sin \psi u \\
& +(\sin \phi \sin \theta \sin \psi+\cos \phi \cos \psi) v \\
& +(\cos \phi \sin \theta \sin \psi-\sin \phi \cos \psi) w \\
\dot{z}= & -\sin \theta u+\sin \phi \cos \theta v+\cos \phi \cos \theta w \\
\dot{\phi}= & p+(\sin (\phi) q+\cos (\phi) r) \tan (\theta) \\
\dot{\theta}= & \cos (\phi) q-\sin (\phi) r \\
\dot{\psi}= & (\sin (\phi) q+\cos (\phi) r) \cos (\theta)-1 \\
I_{x x} \dot{p}=\quad & -l_{b} k_{T}\left(\omega_{1}^{2} \cos \beta_{1}-\omega_{3}^{2} \cos \beta_{3}\right)-\left(I_{z z}-I_{y y}\right) r q \\
& -q I_{r}\left(\omega_{1} \cos \beta_{1}+\omega_{2}+\omega_{3} \cos \beta_{3}+\omega_{4}\right) \\
I_{y y} \dot{q}=\quad & l_{b} k_{T}\left(\omega_{2}^{2}-\omega_{4}^{2}\right)-\left(I_{x x}-I_{z z}\right) r p \\
& -r I_{r}\left(\omega_{1} \sin \beta_{1}+\omega_{3} \sin \beta_{3}\right) \\
& +p I_{r}\left(\omega_{1} \cos \beta_{1}+\omega_{2}+\omega_{3} \cos \beta_{3}+\omega_{4}\right) \\
& +k_{M}\left(\omega_{3}^{2} \sin \beta_{3}-\omega_{1}^{2} \sin \beta_{1}\right) \\
& -l_{b} k_{T}\left(\omega_{1}^{2} \sin \beta_{1}-\omega_{3}^{2} \sin \beta_{3}\right)-\left(I_{y y}-I_{x x}\right) p q \\
& +q I_{r}\left(\omega_{1} \sin \beta_{1}+\omega_{3} \sin \beta_{3}\right) \\
& +k_{M}\left(\omega_{3}^{2} \sin \beta_{3}+\omega_{4}^{2}-\omega_{1}^{2} \sin \beta_{1}-\omega_{2}^{2}\right) \\
I_{z z} \dot{r}= &
\end{aligned}
$$

$$
\begin{gathered}
b(t)=\left[\begin{array}{c}
-g \sin \theta-\frac{k_{T}}{m}\left(\omega_{1}^{2} \sin \beta_{1}+\omega_{3}^{2} \sin \beta_{3}\right) \\
g \sin \phi \cos \theta \\
g \cos \phi \cos \theta-\frac{k_{T}}{m}\left(\omega_{1}^{2} \cos \beta_{1}+\omega_{2}^{2}+\omega_{3}^{2} \cos \beta_{3}+\omega_{4}^{2}\right)
\end{array}\right] \\
y(t)=\dot{\nu}_{1}=\left[\begin{array}{c}
\dot{u} \\
\dot{v} \\
\dot{w}
\end{array}\right]
\end{gathered}
$$

Consider the cascaded filters with matrix states $M \in \mathbb{R}^{3} \times$ $\mathbb{R}^{3}, Q \in \mathbb{R}^{3} \times \mathbb{R}^{3}$, with arbitrary initial conditions such that $\|Q(0)\|>0$, defined as : 


$$
\begin{aligned}
& \dot{M}=-\alpha M+A(t) \\
& \dot{Q}=-\beta Q+M^{T} M
\end{aligned}
$$

where $\alpha \in \mathbb{R}^{+}, \beta \in \mathbb{R}^{+}$are tuning parameters chosen by the designer, along with the two vector systems with state $\rho \in \mathbb{R}^{3}, \delta \in \mathbb{R}^{3}$

$$
\begin{gathered}
\dot{\rho}=-\alpha \rho+\dot{\nu}_{1}-b(t)+M \dot{\nu}_{1} \\
\dot{\delta}=-\beta \delta+\rho \triangleq-\beta \delta+\tilde{z}+M \nu_{1}
\end{gathered}
$$

with arbitrary initial conditions. Notice that the vector $(\rho-$ $\left.M \nu_{1}\right)$ exponentially converges to zero. In fact, by setting $\tilde{z}=\rho-M \nu_{1}$, we have

$$
\dot{\tilde{z}}=-\alpha\left(\rho-M \nu_{1}\right)=-\alpha \tilde{z}
$$

We introduce an estimate of the linear velocity

$$
\hat{\nu}_{1}=\left[\begin{array}{c}
\hat{u} \\
\hat{v} \\
\hat{w}
\end{array}\right]
$$

along with a filter vector state $\xi \in \mathbb{R}^{3}$ that satisfy the differential equations :

$$
\begin{gathered}
\dot{\xi}=-\beta \xi+Q \dot{\nu}_{1}-\dot{M}^{T} \delta \\
\dot{\hat{\nu}}_{1}=\gamma\left(-Q \hat{\nu}_{1}+M^{T} \delta+\xi\right)+\dot{\nu}_{1}
\end{gathered}
$$

with $\gamma \in \mathbb{R}^{3}$ tuning parameter to be chosen by the designer

By setting the estimation error variables $\tilde{\nu}_{1} \triangleq \nu_{1}-\hat{\nu}_{1}$ and $\chi \triangleq Q \nu_{1}-M^{T} \delta-\xi$, we obtain :

$$
\begin{gathered}
\dot{\chi}=-\beta \chi-M^{T} \tilde{z} \\
\dot{\tilde{\nu}}_{1}=\gamma\left(-Q \tilde{\nu}_{1}+\chi\right)
\end{gathered}
$$

From (14) along with (10) and (13), we will show that exponential convergence to zero of estimation error is guaranteed if matrix $\mathrm{Q}$ is positive definite. This is a consequence of the following assumption.

Hypothesis 1: There are positive integers $T, K_{1}^{*}$ and $K_{2}^{*}$ such that

$$
K_{1}^{*} I \geq \int_{t}^{t+T} A(\tau)^{T} A(\tau) d \tau \geq K_{2}^{*} I \quad \text { for all } t \in \mathbf{R}
$$

Remark 1: Hypothesis 1 is a mathematical formulation of the properties that $\mathrm{A}(\mathrm{t})$ is bounded and persistently exciting $(\mathrm{PE})$. The first property is a natural requirement. The second one can be shown to be guaranteed if the UAV tracks a trajectory which is in practice a small periodic orbit. Besides, the nature of the airship provides vibrations in real applications, that may already give enough persistency of excitation).
Previous arguments can be summarized in the following

Proposition 1: Consider the UAV model described by (1)-(4). Under Hypothesis 1 , if the variables $\eta_{2}, \nu_{2}, \dot{\nu}_{1}$ are available for measurement, the dynamic observer described by (6), (7), (8), (9) with state $M(t) \in \mathbb{R}^{3} \times \mathbb{R}^{3}, Q(t) \in$ $\mathbb{R}^{3} \times \mathbb{R}^{3}, \rho(t) \in \mathbb{R}^{3}, \delta(t) \in \mathbb{R}^{3}, \hat{\nu}_{1}(t) \in \mathbb{R}^{3}$, is such that the vector $\nu_{1}-\hat{\nu}_{1}$ converges to zero globally exponentially for any initial conditions $M(0) \in \mathbb{R}^{3} \times \mathbb{R}^{3}, Q(0) \in \mathbb{R}^{3} \times \mathbb{R}^{3}$, $\rho(0) \in \mathbb{R}^{3}, \delta(0) \in \mathbb{R}^{3}, \hat{\nu}_{1}(0) \in \mathbb{R}^{3}$ with $|Q(0)|>0$.

Proof: The stability proof is standard and follows Lyapunov techniques. Consider the Lyapunov function :

$$
V=\frac{1}{2} \kappa_{1} \tilde{\nu}_{1}^{T} \tilde{\nu}_{1}+\frac{1}{2} \kappa_{2} \chi^{T} \chi+\frac{1}{2} \kappa_{3} \tilde{z}^{T} \tilde{z}
$$

By computing the time derivative $\dot{V}$ and recalling (10), (13), (14) we obtain

$$
\begin{aligned}
\dot{V} & =-\alpha \kappa_{3}\|\tilde{z}\|^{2}-\beta \kappa_{2}\|\chi\|^{2}-\kappa_{2} \chi^{T} M^{T} \tilde{z} \\
& -\gamma \kappa_{1} \tilde{\nu}_{1}^{T} Q \tilde{\nu}_{1}+\gamma \kappa_{1} \tilde{\nu}_{1}^{T} \chi
\end{aligned}
$$

Defining the constants $\left|\lambda_{\max }(M)\right|=c_{1}$ and $\lambda_{\min }(Q)=$ $c_{2}$ we deduce the following inequalities

$$
\begin{aligned}
\kappa_{2} \chi^{T} M^{T} \tilde{z} & \leq \frac{\kappa_{2} \beta}{4}\|\chi\|^{2}+\frac{\kappa_{2}}{\beta} c_{1}^{2}\|\tilde{z}\|^{2} \\
\gamma \chi^{T} \tilde{\nu}_{1} & \leq \frac{\gamma c_{2}}{2}\left\|\tilde{\nu}_{1}\right\|^{2}+\frac{\gamma}{2 c_{2}}\|\chi\|^{2}
\end{aligned}
$$

that substituted in (16) yield

$$
\begin{aligned}
\dot{V} & \leq-\left(\kappa_{3} \alpha-\frac{\kappa_{2}}{\beta} c_{1}^{2}\right)\|\tilde{z}\|^{2}-\left(\kappa_{2} \beta-\frac{\kappa_{2} \beta}{4}+\frac{\kappa_{1} \gamma}{2 c_{2}}\right)\|\chi\|^{2} \\
& -\kappa_{1} \gamma\left(c_{2}-\frac{c_{2}}{2}\right)\left\|\tilde{\nu}_{1}\right\|^{2}
\end{aligned}
$$

By setting

$$
\begin{aligned}
\kappa_{3} & =1 \\
\kappa_{2} & =\frac{2 \gamma}{\beta c_{2}} \\
\kappa_{1} & =\frac{2 \kappa_{2} c_{1}^{2}}{\beta \alpha}=\frac{4 \gamma c_{1}^{2}}{\beta^{2} \alpha c_{2}}
\end{aligned}
$$

we finally obtain

$$
\dot{V} \leq-\left(\frac{2 \gamma c_{1}^{2}}{\beta^{2} c_{2}}\right)\|\tilde{z}\|^{2}-\left(\frac{\gamma}{2 c_{2}}\right)\|\chi\|^{2}-\left(\frac{\gamma c_{2}}{2}\right)\left\|\tilde{\nu}_{1}\right\|^{2}
$$

Previous inequality implies that there exist a suitable positive real constant $\lambda$ such that $\dot{V} \leq-\lambda V$, which guarantees the convergence of all errors exponentially to zero.

Simulations were carried out to illustrate the performance of the observer, as well as to verify the effects of the design parameters (see sectionV). Furthermore, simulations were made searching insights on the effect of noisy measurements 
on the observer system. These simulations were the motivation for the development of new schemes aiming a better performance in the presence of noise.

\section{Robust OBSERVER}

In order to quantify the observer performance in case of measurement noise applied on the acceleration signals, we propose a modification on the previous observer. We assume now that a disturbed acceleration $a_{\mu}$ is available ; $a_{\mu} \triangleq \dot{\nu}_{1}+$ $\mu$, where the vector $\mu \in \mathbb{R}^{3}$ represents the measurement noise such that (defining $\mu_{M} \in \mathbb{R}^{+}$) :

$$
\|\mu\| \leq \mu_{M}
$$

Consider the cascaded filters with matrix states $M_{\mu} \in$ $\mathbb{R}^{3} \times \mathbb{R}^{3}, Q_{\mu} \in \mathbb{R}^{3} \times \mathbb{R}^{3}$, with arbitrary initial conditions (with $\left|Q_{\mu}(0)\right|>0$ ) defined as :

$$
\begin{gathered}
\dot{M}_{\mu}=-\bar{\alpha} M_{\mu}+A(t) \\
\dot{Q}_{\mu}=-\beta Q_{\mu}-\frac{k}{4} Q_{\mu} Q_{\mu}^{T} Q_{\mu}+M_{\mu}^{T} M_{\mu}
\end{gathered}
$$

where $\bar{\alpha} \in \mathbb{R}^{+}$is such that $\bar{\alpha}=\alpha+\frac{k}{4}, \beta \in \mathbb{R}^{+}$are tuning parameters chosen by the designer, along with the two vector systems with state $\rho_{\mu} \in \mathbb{R}^{3}, \delta_{\mu} \in \mathbb{R}^{3}$

$$
\begin{aligned}
\dot{\rho}_{\mu} & =-\bar{\alpha} \rho_{\mu}+A(t) \nu_{1}+\mu+M_{\mu} a_{\mu} \\
& =-\bar{\alpha} \rho_{\mu}+a_{\mu}-b(t)+M_{\mu} a_{\mu} \\
\dot{\delta_{\mu}} & =-\beta \delta_{\mu}+\rho_{\mu} \\
& \triangleq-\beta \delta_{\mu}+\tilde{z}_{\mu}+M_{\mu} \nu_{1}
\end{aligned}
$$

with arbitrary initial conditions.

In fact, by setting $\tilde{z}_{\mu}=\rho_{\mu}-M_{\mu} \nu_{1}$, we now have :

$$
\dot{\tilde{z}}_{\mu}=-\bar{\alpha} \tilde{z}_{\mu}+\left(M_{\mu}+I\right) \mu
$$

We introduce an estimate of the linear velocity

$$
\hat{\nu}_{1}=\left[\begin{array}{c}
\hat{u} \\
\hat{v} \\
\hat{w}
\end{array}\right]
$$

along with a filter vector state $\xi_{\mu} \in \mathbb{R}^{3}$ that satisfy the differential equations :

$$
\begin{gathered}
\dot{\xi_{\mu}}=-\beta \xi_{\mu}-\frac{k}{4} Q_{\mu} Q_{\mu}^{T} \xi+Q_{\mu} a_{\mu}-\dot{M}_{\mu}{ }^{T} \delta_{\mu}-\frac{k}{4} Q_{\mu} Q_{\mu}^{T} M_{\mu}^{T} \delta_{\mu} \\
\dot{\hat{\nu}}_{1}=\gamma\left(-Q_{\mu} \hat{\nu}_{1}+M_{\mu}^{T} \delta_{\mu}+\xi_{\mu}\right)+a_{\mu}
\end{gathered}
$$

with $\gamma \in \mathbb{R}^{3}$ tuning parameter to be chosen by the designer

By setting the estimation error variables $\tilde{\nu}_{1} \triangleq \nu_{1}-\hat{\nu}_{1}$ and $\chi_{\mu} \triangleq Q \nu_{1}-M_{\mu}^{T} \delta_{\mu}-\xi_{\mu}$, we obtain :

$$
\begin{gathered}
\dot{\chi_{\mu}}=-\left(\beta+\frac{k}{4} Q_{\mu} Q_{\mu}^{T}\right) \chi_{\mu}-M_{\mu}^{T} \tilde{z}_{\mu}-Q_{\mu} \mu \\
\dot{\tilde{\nu}}_{1}=\gamma\left(-Q_{\mu} \tilde{\nu}_{1}+\chi_{\mu}\right)-\mu
\end{gathered}
$$

Proposition 2: Consider the UAV model described by (1)-(4). Under Hypothesis 1 , if the variables $\eta_{2}, \nu_{2}, a_{\mu}$ are available for measurement, the dynamic observer described by (18), (19), (20), (21) with state $M_{\mu}(t) \in \mathbb{R}^{3} \times \mathbb{R}^{3}$, $Q_{\mu}(t) \in \mathbb{R}^{3} \times \mathbb{R}^{3}, \rho_{\mu}(t) \in \mathbb{R}^{3}, \delta_{\mu}(t) \in \mathbb{R}^{3}, \hat{\nu}_{1}(t) \in \mathbb{R}^{3}$, is such that for any given $\epsilon>0$, there exist a suitable combination of the tuning parameters yelding $\mathcal{L}_{2}$ and $\mathcal{L}_{\infty}$ gains from the measurement error $\mu$ to the estimations error $\left(\nu_{1}-\hat{\nu}_{1}\right)$ smaller than $\epsilon$, for any initial conditions $M_{\mu}(0) \epsilon$ $\mathbb{R}^{3} \times \mathbb{R}^{3}, Q_{\mu}(0) \in \mathbb{R}^{3} \times \mathbb{R}^{3}, \rho_{\mu}(0) \in \mathbb{R}^{3}, \delta_{\mu}(0) \in \mathbb{R}^{3}$, $\hat{\nu}_{1}(0) \in \mathbb{R}^{3}$ with $\left|Q_{\mu}(0)\right|>0$.

Proof: The stability proof follows the same method applied before. Consider the Lyapunov function

$$
V=\frac{1}{2} \kappa_{1} \tilde{\nu}_{1}^{T} \tilde{\nu}_{1}+\frac{1}{2} \kappa_{2} \chi_{\mu}^{T} \chi_{\mu}+\frac{1}{2} \kappa_{3} \tilde{z}_{\mu}^{T} \tilde{z}_{\mu}
$$

By computing the time derivative of $V$, we have :

$$
\begin{aligned}
\dot{V} & =-\kappa_{1} \gamma \tilde{\nu}_{1}^{T} Q_{\mu} \tilde{\nu}_{1}-\kappa_{2} \beta \chi_{\mu}^{T} \chi_{\mu}-\kappa_{2} \frac{k}{4} \chi_{\mu}^{T} Q_{\mu} Q_{\mu}^{T} \chi_{\mu} \\
& -\kappa_{3}\left(\alpha+\frac{k}{4}\right) \tilde{z}_{\mu}^{T} \tilde{z}_{\mu}+\kappa_{1} \gamma \tilde{\nu}_{1}^{T} \chi_{\mu}-\kappa_{2} \chi_{\mu}^{T} M_{\mu}^{T} \tilde{z}_{\mu} \\
& -\kappa_{1} \tilde{\nu}_{1}^{T} \mu-\kappa_{2} \chi_{\mu}^{T} Q_{\mu} \mu+\kappa_{3} \tilde{z}_{\mu}^{T}\left(M_{\mu}+I\right) \mu
\end{aligned}
$$

As in the previous scheme, matrix $Q_{\mu}$ must be positive definite. Defining now the constants $\left|\lambda_{\max }\left(M_{\mu}\right)\right|=c_{1}$, $\lambda_{\min }\left(Q_{\mu}\right)=c_{2}$, we obtain :

$$
\begin{aligned}
\dot{V} \leq & -\kappa_{1} \gamma c_{2}\left\|\tilde{\nu}_{1}\right\|^{2}-\kappa_{2} \beta\left\|\chi_{\mu}\right\|^{2}-\kappa_{3} \alpha\left\|\tilde{z}_{\mu}\right\|^{2} \\
& +\kappa_{1} \gamma \frac{c_{2}}{2}\left\|\tilde{\nu}_{1}\right\|^{2}+\kappa_{1} k\left\|\tilde{\nu}_{1}\right\|^{2}+\frac{\kappa_{1} \gamma}{2 c_{2}}\left\|\chi_{\mu}\right\|^{2} \\
& +\frac{\kappa_{2} \beta}{4}\left\|\chi_{\mu}\right\|^{2}+\frac{c_{1}^{2} \kappa_{2}}{\beta}\left\|\tilde{z}_{\mu}\right\|^{2}+\frac{\kappa_{3}}{k}\left(c_{1}+1\right)^{2}\|\mu\|^{2} \\
& +\frac{\kappa_{2}}{k}\|\mu\|^{2}+\frac{\kappa_{1}}{k}\|\mu\|^{2}
\end{aligned}
$$

By choosing :

$$
\begin{aligned}
\kappa_{1} & =\frac{1}{\gamma} \\
\kappa_{2} & =\frac{2}{\beta c_{2}} \\
\kappa_{3} & =\frac{2 \kappa_{2} c_{1}^{2}}{\beta \alpha}=\frac{4 c_{1}^{2}}{\beta^{2} \alpha c_{2}}
\end{aligned}
$$

and by setting the design condition $\gamma>\frac{2 k}{c_{2}}$, we may finally state that for a suitable $\alpha_{i} \in \mathbb{R}^{+}$, one may rewrite equation (28) as :

$$
\dot{V} \leq-\alpha_{1}\left\|\tilde{\nu}_{1}\right\|^{2}-\alpha_{2}\left\|\chi_{\mu}\right\|^{2}-\alpha_{3}\left\|\tilde{z}_{\mu}\right\|^{2}+\frac{\alpha_{4}}{k}\|\mu\|^{2}
$$


which guarantees arbitrary $\mathcal{L}_{\infty}$ and $\mathcal{L}_{2}$ robustness from the measurement error $\mu$ to the estimation errors $\tilde{\nu}_{1}, \chi_{\mu}$ and $\tilde{z}_{\mu}$

\section{Simulation Results}

In this section we illustrate the observer designed above to estimate the linear velocity of an UAV based on the measurable angles, angular velocities and linear accelerations. These simulations also illustrate the effect of the tuning parameters $\alpha, \beta$ and $\gamma$ on the observer performances. In all simulations the observer estimations have initial conditions set to zero, while the desired states are time varying (and different from zero at $t=0$ ). In all simulations both observers track the desired time varying states, with different performances and noise rejections. We have used the following parameters values :

$$
\begin{array}{ll}
m=2.500 \mathrm{Kg} & k_{T}=10^{-5} \mathrm{~N} . \mathrm{s}^{2} \\
l_{b}=23 \mathrm{~cm} & I_{R}=10010^{-7} \mathrm{Kgm}^{2} \\
I_{x x}=22493110^{-7} \mathrm{Kgm}^{2} & I_{y y}=22261110^{-7} \mathrm{Kgm}^{2} \\
I_{z z}=32513010^{-7} \mathrm{Kgm}^{2} & k_{M}=910^{-5} \mathrm{Ns}^{2} \mathrm{~m} \\
u_{G}=0.032 \mathrm{~m} &
\end{array}
$$

Consider the first version of the observer, that does not take into account the measurement noise. The time history of the three speeds to be observed is shown in Fig. 2a, and observer estimates in Fig. 2b, while estimation errors going exponentially to zero are shown in Fig. 2c. In Fig. 3 the angular speeds, the angles, and the accelerations are plotted. Small periodic orbits were considered in order to satisfy the persistency of excitation condition.
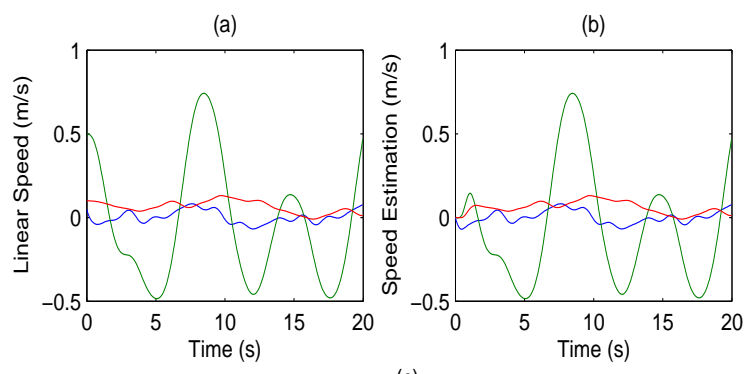

(c)

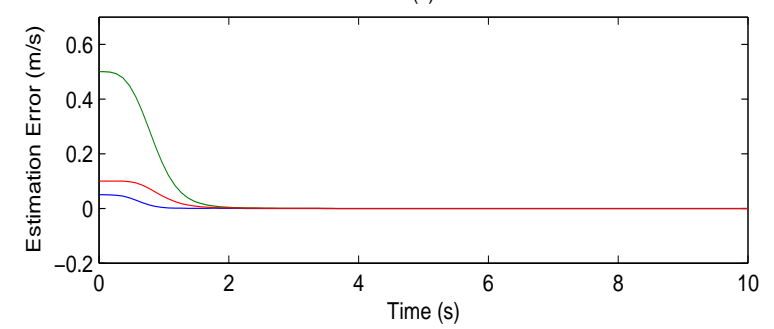

Fig. 2. (a) Linear Speed, (b) Speed Estimation, (c) Estimation Error

We have considered the first observer in the case of additive measurement noise using. The measured acce- leration is presented in Fig. 4 where the noise $\mu$ is 10 percent of the measured acceleration. One may see in Fig. $5 \mathrm{a}$ the velocity estimated by the observer, while Fig. 5b describes the estimation time history, and Fig. 5c illustrates the observation error going exponentially to a residual set given by the noise amplitude.

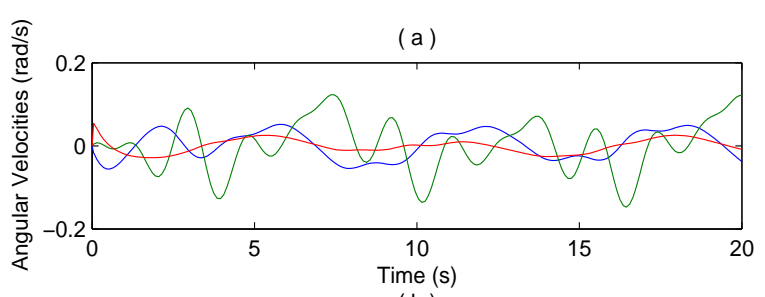

(b)

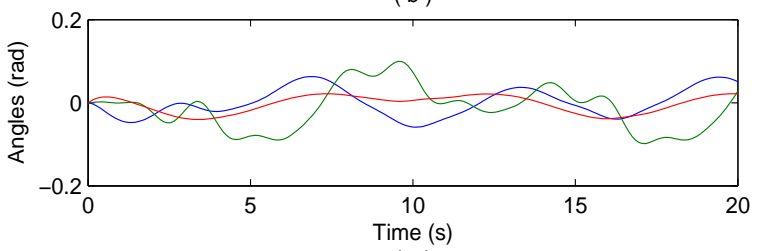

(c)

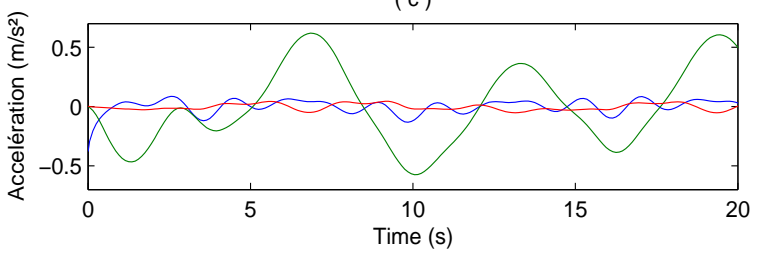

Fig. 3. (a) Angular Speed, (b) Angles, (c) Acceleration

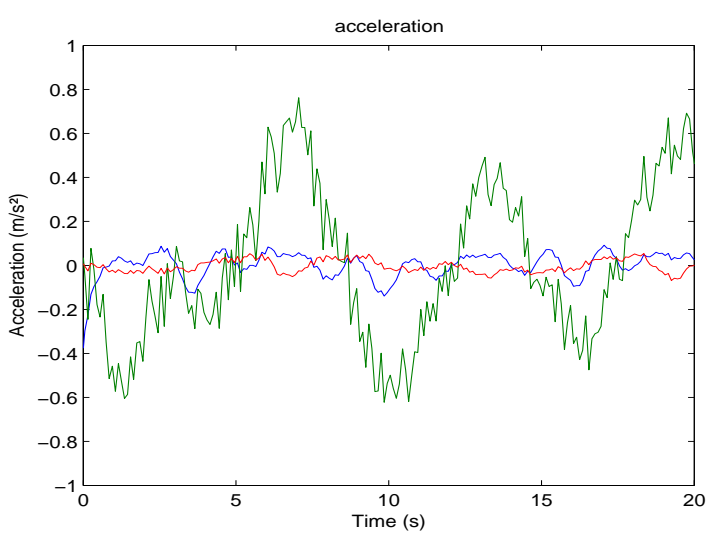

Fig. 4. Acceleration

The same simulation is undertaken using the second version of the observer, where the disturbance is attenuated following the design parameter $k$. To describe the effect of this parameter, we present three simulations using different values of $k$. The real speed and the measurement noise are the same presented in Fig. 5 a and b, while in Fig. 6 we show the results of the simullations using $k=0, k=10$ and $k=50$. The main effects of larger values of $k$ are 
a greater attenuation, in trend of a slower convergence rate. These results are in accordance with our claims, and illustrate the mechanisms of the tuning procedure.

(a)
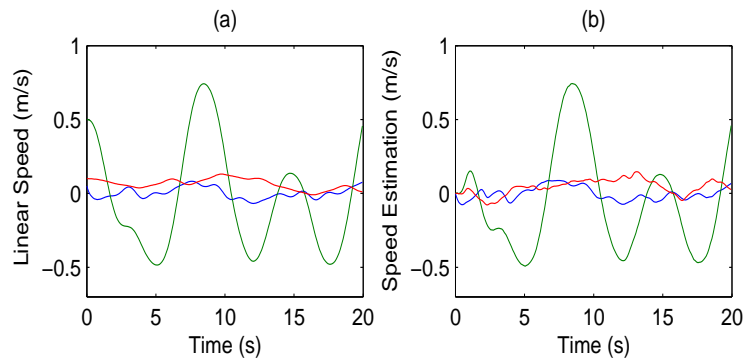

(c)

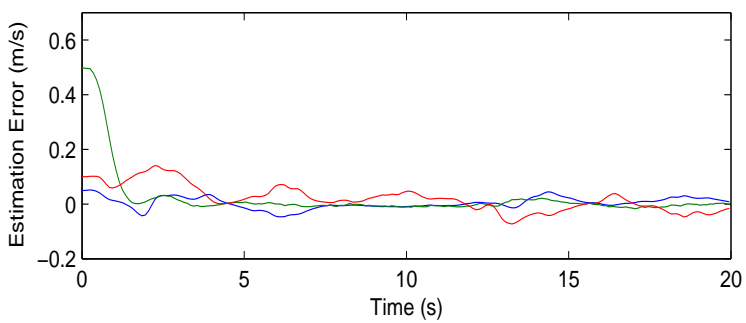

Fig. 5. (a) Linear Speed, (b) Speed Estimation, (c) Estimation Error

(a)

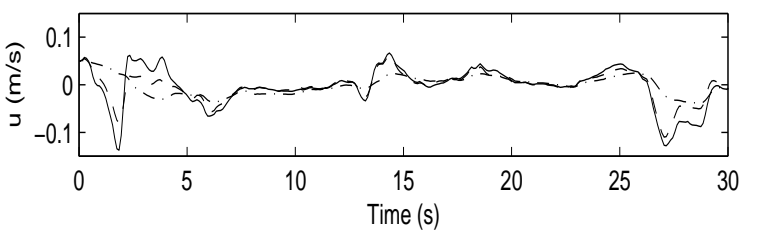

(b)

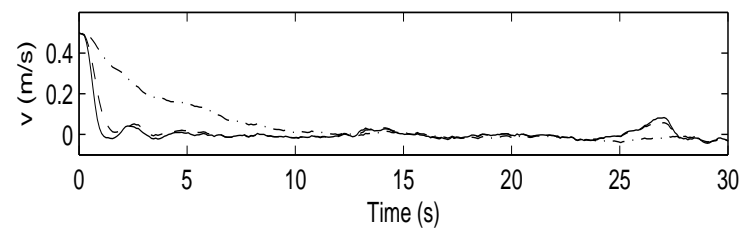

(c)

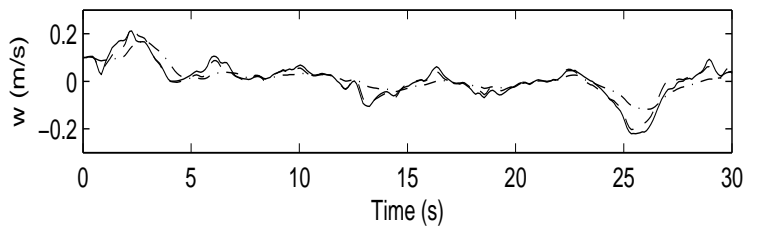

Fig. 6. Estimated values of $\mathrm{u}$ in (a), $\mathrm{v}$ in (b) and $\mathrm{w}$ in (c) depending on

\section{CONCLUSION}

In this work, the classical problem of speed estimation of an Unmanned Aerial Vehicle has been adressed when the acceleration, the angles and the angular speeds are available for measurement. A solution has been provided for a class of systems via the tools of adaptive observation theory with promissing results. Future research directions will focus on executing experiments on the physical prototype, along with relaxing the condition of persistency of excitation used to prove exponential convergence. Complementary work will also be done in the direction of faster convergence of parameters, as well as a better independence from exponential convergence rate, and noise attenuation.

\section{REFERENCES}

[1] R. Marino, P. Tomei, « Nonlinear Control Design - Geometric, Adaptive and Robust », Prentice Hall, Hemel Hempstead, London, 1995.

[2] R. Marino, G. L. Santossuosso, P. Tomei, Robust Adaptive Observers for Nonlinear Systems with Bounded Disturbances, IEEE Transactions on automatic control, Vol. 46, No 6, pp 967-972, June 2001.

[3] H. Khalil, « Nonlinear Systems » $3^{\text {rd }}$ edition , Prentice Hall, New Jersey, 1996.

[4] Naoufel Azouz, «Modele dynamique du microdrone XSF ». Laboratoire IBISC CNRS/Universite d'Evry Val d'Essonne, internal report (in french).

[5] R. Lozano, A. Dzul, P. Castillo. Global stabilization of the PVTOL : Real-time application to a mini-aircraft, vol. 77, number 8, pp. 735740, May 2004.

[6] P. Castillo, A. Dzul, R. Lozano. Real-time stabilization and tracking of a four-rotor mini rotorcraft, IEEE Transaction on control systems technology, vol. 12, pp. 510-516, July 2004.

[7] N. Guenardt, T. Hamel, V. Moreaut. Dynamic modeling and intuitive control strategy for an "X4-flyer", International Conference on Control and Automation (ICCA2005), June 27-29, 2005, Budapest, Hungary MM-4.4.

[8] P. Pounds, R. Mahony, P. Hynes, J. Roberts, Design of a FourRotor Aerial Robot, Procedings of 2002 Australasian Conference on Robotics and Automation, Auckland, 27-29 November 2002.

[9] K. Benzemrane «Système Observateur pour un drone hélicoptère » . Laboratoire IBISC - CNRS / Universite d'Evry Val d'Essonne (in french). Juin 2006.

[10] P. Castillo, P. Albertos, P. Garcia, R. Lozano. Simple Real-time Attitude Stabilization of a Quad-rotor Aircraft With Bounded Signals, Proceedings of the 45th IEEE Conference on Decision \& Control, San Diego, CA, USA, December 13-15, 2006, December 13-15, 2006.

[11] F. Kendoul, D. Lara, I. Fantoni, R. Lozano. Nonlinear control for systems with bounded inputs : Real-time embedded control applied to UAVs, Proceedings of the 45th IEEE Conference on Decision \& Control, San Diego, CA, USA, December 13-15, 2006, December 13$15,2006$.

[12] T. Madani and A. Benallegue. Control of a Quadrotor Mini-Helicopter via Full State Backstepping Technique, Proceedings of the 45th IEEE Conference on Decision \& Control, San Diego, CA, USA, December 13-15, 2006 December 13-15, 2006.

[13] B. Vik, T.I. Fossen. A nonlinear observer for GPS and INS integration, Proceedings of the 40th IEEE Conference on Decision and Control, Orlando, Florida, USA December 2001.

[14] Y. Zhao, J.J. Slotine. Discrete nonlinear observers for inertial navigation. Systems and Control Letters, 2005. 